CLINICAL STUDY

\title{
Effectiveness of eplerenone or spironolactone treatment in preserving renal function in primary aldosteronism
}

Verena Fourkiotis, Oliver Vonend ${ }^{1}$ Sven Diederich ${ }^{2}$, Evelyn Fischer ${ }^{3}$, Katharina Lang ${ }^{4}$, Stephan Endres ${ }^{3}$ Felix Beuschlein ${ }^{3}$, Holger S Willenberg ${ }^{5}$, Lars C Rump ${ }^{1}$, Bruno Allolio ${ }^{4}$, Martin Reincke ${ }^{3}$, Marcus Quinkler and for the Mephisto Study Group

Clinical Endocrinology. Charité Campus Mitte, Charité University Medicine Berlin, Charitéplatz 1, D 10117 Berlin, Germany. ${ }^{1}$ Department of Nephrology, Medical Faculty, Heinrich-Heine University Duesseldorf, Duesseldorf, Germany, ${ }^{2}$ Endokrinologikum Berlin, Berlin, Germany, ${ }^{3}$ Department of Endocrinology and Metabolism, Medizinische Klinik - Innenstadt, University Hospital Munich, Munich, Germany, ${ }^{4}$ Endocrinology and Diabetes Unit, Department of Internal Medicine I, University Hospital of Wuerzburg, Wuerzburg, Germany and ${ }^{5}$ Department of Endocrinology, Diabetes and Rheumatology, Medical Faculty, Heinrich-Heine University Duesseldorf, Duesseldorf, Germany

(Correspondence should be addressed to M Quinkler; Email: marcus.quinkler@charite.de)

\begin{abstract}
Objective: Primary aldosteronism (PA) has deleterious effects on kidney function independent of blood pressure levels. Up to now, data on effectiveness of different PA therapies regarding renal function are scarce.

Design and methods: This prospective multi-center study included 29 patients with newly diagnosed PA evaluated before and 1 year after treatment initiation, and a second cohort including 119 patients who were evaluated 5.3 and 6.8 years after treatment initiation. Glomerular filtration rate (GFR), spot urine albumin excretion/urinary creatinine (UAE/Ucrea) ratio, biochemical parameters, and 24-h blood pressure were measured. In a larger cross-sectional cohort, renal function was evaluated depending on the type of treatment (adrenalectomy (ADX; $n=86)$; spironolactone $(n=65)$; and eplerenone $(n=18))$.

Results: GFR and UAE/Ucrea ratio significantly decreased in newly diagnosed PA patients after treatment initiation. In the second cohort, GFR and UAE/Ucrea ratio did not change during study period, and blood pressure was well controlled. In the larger cross-sectional cohort, no differences were seen in GFR and UAE/Ucrea ratio between PA patients on different treatment regimens. However, eplerenone treatment showed lower potassium levels and higher number of required antihypertensive medications.

Conclusions: Renal dysfunction with elevated albuminuria was seen in PA patients and was reversible after treatment initiation. Medical therapies with spironolactone or eplerenone seem to be as effective as ADX regarding renal function and blood pressure; however, sufficient daily doses need to be given.
\end{abstract}

European Journal of Endocrinology 168 75-81

\section{Introduction}

Primary aldosteronism (PA) is the most common form of secondary hypertension and affects up to $10 \%$ of all hypertensive patients $(1,2,3,4,5)$. Patients with PA have an increased risk of developing relevant comortalities and comorbidities, e.g. vascular, cardiac or cerebrovascular morbidities, and renal insufficiency $(2,6,7,8)$. On the basis of the German Conn's Registry, we have discovered a positive correlation between aldosterone levels and the prevalence of the most common comorbidities, including the prevalence of chronic renal failure (2). There is strong evidence that autonomous aldosterone secretion causes an earlier and more extensive renal damage than essential hypertension $(\mathrm{EH})(5,9,10)$. The excess of proteinuria, an early marker of renal damage, is significantly higher in patients with PA compared with patients with $\mathrm{EH}$ $(9,11)$. The Italian PAPY study reported an even stronger effect on urinary albumin excretion in patients with aldosterone-producing adenoma (APA) than in those with bilateral adrenal hyperplasia (BAH) (5). Treatments of PA are effective in correcting hypertension and hypokalemia, and currently available information on their capability to prevent cardiovascular events and deterioration of renal function indicates that surgery and medical treatment are equally beneficial in the long-term $(12,13)$. Recent studies have suggested different pathomechanisms with a direct harmful effect of aldosterone on kidney tissue. In a clinical study, human kidney biopsies from patients with proteinuria, hematuria, or deterioration of kidney function for uncertain reasons were investigated, showing a highly upregulated mineralocorticoid receptor (MR) expression 
and upregulated inflammatory markers in the group with severe proteinuria compared with those without proteinuria (14). Further clinical and experimental studies have demonstrated an increased level of inflammatory markers such as macrophage-chemoattractant protein 1 and transforming growth factor $\beta 1$, osteopontin, interleukin 1 (IL1), and IL6 expression due to aldosterone excess $(14,15,16,17,18)$. Especially, the excess of aldosterone in combination with an elevated salt intake results in renal inflammation, fibrosis, podocyte injury, and mesangial cell proliferation $(19,20)$.

In summary, aldosterone excess has deleterious effects on kidney function independent of blood pressure levels. Therefore, diagnosis of PA is important and treatment of PA should not only aim at lowering blood pressure but also at prevention of morbidity and mortality, especially renal impairment.

Today, bilateral disease in PA is treated with MR antagonists while patients with unilateral PA usually undergo adrenalectomy (ADX) (1). Both treatment regimens are thought to abolish the risk of increased comorbidities and comortalities. However, up to now data regarding the effectiveness of different PA treatment forms on renal function are scarce (10), especially concerning the more selective MR antagonist eplerenone. We, therefore, analyzed prospective data of the German Conn's Registry to compare the effectiveness of different treatment forms on kidney function. The specific questions were as follows:

i) What is the effect of therapy initiation on glomerular filtration rate (GFR) and urinary albumin excretion in newly diagnosed PA patients and in treated PA patients within the long-term follow-up?

ii) Are there differences regarding renal function between patients treated with ADX compared with those treated with the MR antagonists spironolactone or eplerenone?

\section{Materials and methods}

\section{Design and patients}

The German Conn's Registry (www.conn-register.de) is a multi-center database analyzing comorbidities and long-term outcome of patients with PA $(2,3,21)$. Since the initiation of the prospective phase in October 2008, all patients actively treated within the centers were entered in a common database after pseudonymization (22). For this study, the patients of the four largest German centers (Munich, $n=146$; Berlin, $n=84$; Düsseldorf, $n=55$; Würzburg, $n=12$ ) treated prospectively between 2008 and August 2011 were included in the analysis. The Ethics Committees of the University of Munich and of the participating centers approved the protocol. Data protection laws were strictly adhered to.
Clinical data at the time of diagnosis were extracted from patients' charts including laboratory test results, initiation of mineralocorticoid antagonist treatment, surgical treatment, cardiovascular comorbidities, BMI, and metabolic conditions. In case of multiple determinations, the measurements of potassium, plasma renin concentration, and aldosterone at first presentation were used for statistical calculations. Blood was generally drawn in fasting state, although this was not standardized within centers. Every 6 and 12 months the patients were seen for follow-up visits including a clinical examination, complete laboratory investigation, cardiovascular examinations, and 24-h urinary sampling. GFR was calculated by the abbreviated MDRD equation: GFR $\left(\mathrm{ml} / \mathrm{min}\right.$ per $\left.1.73 \mathrm{~m}^{2}\right)=186 \times($ serum creatinine/0.95 $)^{-1.154} \times(\text { age })^{-0.203} \times(0.742$ if female) $\times$ (1.21 if colored). In case of significant changes in renal function between groups, eGFR values were reanalyzed by using the CKD-EPI equation. This was done, as the MDRD equation has limitations on eGFR values $>60 \mathrm{ml} / \mathrm{min}$ per $1.73 \mathrm{~m}^{2}(23,24)$.

The diagnostic criteria for PA in this study were chosen according to the Endocrine Society Practice guidelines (25). All patients included had an elevated aldosterone-renin ratio (ARR) and an abnormal confirmatory test (saline infusion test, fludrocortisone suppression test, captopril test, or oral salt loading test with demonstration of elevated excretion of aldosterone and its metabolites in urine) $(2,3)$. Adjustment of medication before screening and confirmation was performed whenever possible, with beta-blockers, central alpha-2 agonists, angiotensin-converting enzyme inhibitors, angiotensin receptor blockers, and diuretics withdrawn for at least 1 week, and mineralocorticoid antagonists at least for 4 weeks. The diagnosis of PA was centrally verified by review of all available data.

\section{Whole cohort}

Two hundred and ninety-seven patients with confirmed PA from the German Conn's Registry for whom sufficient data coverage was available were included in the final analysis. Subtype differentiation between APA and BAH was based on adrenal imaging (computed tomography or magnetic resonance imaging). In addition, adrenal vein sampling was performed in $54-87 \%$ of the patients in the participating centers (26). Unilateral ADX was performed in 127 of 297 patients $(43 \%)$ for suspected unilateral aldosterone excess, mainly APA. The remaining non-operated 170 patients were treated with different medical regimens: spironolactone $(n=80 ; 26 \%)$, eplerenone $(n=22 ; 7 \%)$, or other antihypertensives $(n=68 ; 22 \%)$. For further analysis we distinguished between two patient cohorts in our prospective cohort. 


\section{Prospective cohort of newly diagnosed PA patients}

Newly diagnosed PA patients (since October 2008; cohort 1) were included and evaluated before start of therapy (pretreatment) and followed up thereafter (follow-up). Of the 109 newly diagnosed PA patients, complete data of 29 patients were available including pretreatment and 12-month follow-up visits. Only patients with a complete data set were included.

\section{Prospective cohort of diagnosed PA patients on therapy}

PA patients (diagnosis of PA before October 2008; cohort 2) were included in the study after initiation of therapy had already started, and were evaluated during long-term follow-up at two outpatient visits (called visits V1 and V2, which were $\sim 1.5$ years apart). Of these 188 patients, 119 patients had a complete data set of follow-up visits V1 and V2. The impact of therapy was evaluated in a cross-sectional approach in all 188 patients of this cohort 2 at visit $\mathrm{V} 1$.

\section{Statistical analysis}

Before statistical analysis, parameters were checked for normal distribution. Linear regression analyses were performed to determine correlations between normally distributed data variables. When required, as determined by normality testing for skewness, data variables were normalized by log transformation. The influence of age, disease duration, BMI, potassium, blood pressure, and other parameters on kidney function and urinary albumin excretion was analyzed by linear multivariate regression analysis in a backward-stepwise fashion. Characteristics of the study subjects were compared among groups by the Student's $t$-test. The Pearson's $\chi^{2}$-test was used to compare categorical variables. Changes from pretreatment (cohort 1) or visit V1 (cohort 2) were assessed by twoway ANOVA. Analyses were performed using the statistical software package SPSS, version 15.0 (IBM SPSS Statistics). Significance was accepted if $P<0.05$.

\section{Results}

\section{Cohort 1}

Twenty-nine patients ( 12 women, 17 men) with a mean age of $48.6 \pm 2.1$ years (range 25-66 years) were examined at diagnosis of PA (before treatment initiation) and $1.0 \pm 0.1$ years afterwards under therapy. At diagnosis of PA, the patients had arterial hypertension for $10.1 \pm 1.7$ years. After treatment initiation, the systolic and diastolic blood pressures decreased significantly, especially night time blood pressure values which were lowered considerably (Table 1). Furthermore, potassium levels increased, and aldosterone levels
Table 1 Clinical and biochemical parameters in 29 patients with PA before (pretreatment) and $1.0 \pm 0.1$ years after initiation of treatment (follow-up; cohort 1). Data are means \pm S.E.M.

\begin{tabular}{lcc}
\hline & Pretreatment & Follow-up \\
\hline BMI (kg/m²) & $28.0 \pm 1.1$ & $27.9 \pm 1.0$ \\
Systolic/diastolic BP (mmHg) & $154 / 92 \pm 5 / 2$ & $131^{\ddagger} / 83^{\dagger} \pm 3 / 2$ \\
24-h systolic/diastolic BP & $147 / 90 \pm 4 / 2$ & $134^{\star} / 83^{\star} \pm 4 / 2$ \\
$\quad(\mathrm{mmHg})$ & & \\
24-h systolic/diastolic BP day & $148 / 91 \pm 4 / 2$ & $137 / 85 \pm 4 / 3$ \\
$\quad(\mathrm{mmHg})$ & & \\
24-h systolic/diastolic BP night & $142 / 85 \pm 4 / 2$ & $126^{\star} / 77^{\star} \pm 6 / 3$ \\
$\quad$ (mmHg) & $3.1 \pm 0.3$ & \\
No. of antihypertensive drugs & $141.3 \pm 0.6$ & $2.3 \pm 0.4^{\star}$ \\
Serum sodium (mmol/l) & $3.45 \pm 0.11$ & $139.7 \pm 0.6^{\star}$ \\
Serum potassium (mmol/l) & $264.1 \pm 49.8$ & $4.07 \pm 0.09^{\dagger}$ \\
Aldosterone (ng/l) & $67.8 \pm 13.4$ & $43.7 \pm 17.0^{\dagger}$ \\
ARR & $17.8 \pm 2.0$ & $13.8 \pm 7.2^{\dagger}$ \\
Urea (mg/dl) & $0.83 \pm 0.05$ & $24.7 \pm 2.8^{\ddagger}$ \\
Serum creatinine (mg/dl) & $92.3 \pm 4.1$ & $0.93 \pm 0.05^{\star}$ \\
GFR (ml/min per 1.73 m ${ }^{2}$ ) & $89.1 \pm 33.0$ & $81.5 \pm 3.8^{\ddagger}$ \\
UAE/UCrea (mg/g) & $19.8 \pm 9.8^{*}$ \\
\hline
\end{tabular}

$\mathrm{BP}$, blood pressure; ARR, aldosterone-renin ratio; UAE/UCrea, spot urine albumin excretion/urinary creatinine. Normal ranges ( $\mathrm{SI}$ units shown in brackets): sodium, 134-145 mmol/l; potassium, 3.4-5.2 mmol/l; ARR, <20; urea, $10-50 \mathrm{mg} / \mathrm{dl}$; UAE/UCrea, $<20 \mathrm{mg} / \mathrm{g} .{ }^{*} P<0.05 ;{ }^{\dagger} P<0.01 ;{ }^{\ddagger} P<0.001$ compared with pretreatment.

and ARR dropped (Table 1). Serum sodium levels decreased and serum urea levels increased significantly (Table 1). The mean GFR decreased significantly after start of treatment (Table 1), as did the spot urine albumin excretion/urinary creatinine (UAE/Ucrea) ratio measured in spot urine samples (Table 1). We analyzed cohort 1 regarding the type of therapy (ADX vs medical therapy; Table 2). The only significant difference between the ADX group and the medical therapy group before treatment initiation was the potassium level $(3.25 \pm 0.51$ and 3.83 $\pm 0.65 \mathrm{mmol} / \mathrm{l}$ respectively; $P<0.05)$. After 1 year of treatment, medical therapy (spironolactone 55.0 $\pm 7.3 \mathrm{mg} /$ day) was not as effective as ADX in lowering systolic blood pressure and increasing potassium levels; however, UAE/Ucrea ratio was not different.

Stepwise multiple regression analyses found age to be the only significant predictor for GFR $(P<0.001$, $R=-0.736$ ) in cohort 1 . For UAE/Ucrea ratio no significant predictors were found.

\section{Cohort 2}

One hundred and nineteen patients (47 women, 72 men) with a mean age of $61.0 \pm 1.0$ years (range 22-83 years) were already on therapy for PA diagnosed $5.3 \pm 0.4$ years before study inclusion (range 0.5-34 years), and were evaluated in a prospective fashion during long-term follow-up. At visit 1, the patients had arterial hypertension for $18.8 \pm 1.1$ years. The clinical and biochemical data are presented in Table 3. During long-term follow-up, patients' blood pressure and potassium levels were within the normal range (Table 3), and GFR and UAE/Ucrea ratio (Table 3) remained stable over the study period ( $16 \pm 0.4$ months). 
Table 2 Clinical and biochemical parameters depending on the therapy in 29 patients with PA at follow-up $1.0 \pm 0.1$ years after initiation of treatment (cohort 1). Data are means \pm S.E.M.

\begin{tabular}{lcc}
\hline & ADX & $\begin{array}{c}\text { Medical } \\
\text { therapy }\end{array}$ \\
\hline$n$ (F/M) & $18(8 / 10)$ & $11(4 / 7)$ \\
Age (years) & $49.4 \pm 2.4$ & $51.7 \pm 4.3$ \\
BMI (kg/m ${ }^{2}$ ) & $27.8 \pm 1.3$ & $28.1 \pm 1.8$ \\
Duration of hypertension (years) & $9.5 \pm 2.0$ & $13.5 \pm 2.9$ \\
Dose MRA (mg/day) & - & $55.0 \pm 7.3$ \\
Systolic/diastolic BP (mmHg) & $126 / 80 \pm 2 / 3$ & $139^{*} / 84 \pm 6 / 3$ \\
24-h systolic/diastolic BP (mmHg) & $130 / 81 \pm 3 / 2$ & $137 / 86 \pm 8 / 3$ \\
24-h systolic/diastolic BP day (mmHg) & $133 / 84 \pm 4 / 3$ & $140 / 88 \pm 7 / 3$ \\
24-h systolic/diastolic BP night & $121 / 74 \pm 4 / 2$ & $132 / 80 \pm 12 / 5$ \\
(mmHg) & & \\
No. of antihypertensive drugs & $2.3 \pm 0.6$ & $2.4 \pm 0.6$ \\
Serum sodium (mmol/l) & $139.8 \pm 0.8$ & $140.5 \pm 1.0$ \\
Serum potassium (mmol/l) & $4.22 \pm 0.10$ & $3.82 \pm 0.18^{\star}$ \\
Aldosterone (ng/l) & $58.3 \pm 9.0$ & $159.3 \pm 35.0^{\dagger}$ \\
ARR & $5.4 \pm 1.1$ & $28.1 \pm 19.1$ \\
Urea (mg/dl) & $25.5 \pm 3.5$ & $22.9 \pm 4.8$ \\
GFR (m//min per 1.73 m ${ }^{2}$ ) & $78.9 \pm 4.6$ & $86.8 \pm 6.6$ \\
UAE/UCrea (mg/g) & $25.5 \pm 13.3$ & $8.9 \pm 1.9$ \\
\hline
\end{tabular}

$\mathrm{ADX}$, adrenalectomy; $\mathrm{BP}$, blood pressure; $\mathrm{ARR}$, aldosterone-renin ratio; UAE/UCrea, spot urine albumin excretion/urinary creatinine. Normal ranges (SI units shown in brackets): sodium, 134-145 mmol/l; potassium, 3.4$5.2 \mathrm{mmol} / \mathrm{l} ; \mathrm{ARR}, \quad<20$; urea, $10-50 \mathrm{mg} / \mathrm{dl}$; UAE/UCrea, $<20 \mathrm{mg} / \mathrm{g}$. ${ }^{*} P<0.05 ;{ }^{\dagger} P<0.01$ compared with ADX.

In a cross-sectional approach, we analyzed the whole of cohort $2(n=188)$ regarding their therapeutic regimen at V1 (Table 4). Most of the patients were adrenalectomized $(n=86)$. Sixty-five patients received spironolactone (daily dose $63.5 \pm 5.8 \mathrm{mg} /$ day), and 18 patients received eplerenone (daily dose 88.2 $\pm 11.0 \mathrm{mg} /$ day). Nineteen patients were treated with other antihypertensive drugs due to side effects of spironolactone. The spironolactone group was older than the other treatment groups. The duration of PA was similar in all treatment groups, whereas the spironolactone group had the longest period of existing hypertension (Table 4). There were no significant differences between the groups regarding ambulatory blood pressure and 24-h blood pressure measurements except that the group without specific anti-mineralocorticoid therapy had significantly higher 24-h systolic blood pressure levels $(P<0.05)$. The group receiving other antihypertensives and the eplerenone group showed the lowest potassium levels. The number of antihypertensive medications was the highest in the eplerenone group. The ADX group had the lowest aldosterone levels and lowest ARR as expected. No differences were seen in the spot urine UAE/Ucrea ratio and the GFR between the groups (Table 4 ).

In cohort 2 , besides higher age $(P<0.001, R=$ $-0.440)$, serum urea levels $(P<0.001, R=-0.595)$ and serum potassium levels $(P<0.001, R=-0.345)$ were also found to be significant predictors of GFR. For UAE/Ucrea ratio no significant predictors were found.

It is well known that gynecomastia is one of the major side effects of spironolactone in men and occurs in a dose-dependent manner with $\sim 7 \%$ of cases with $<50 \mathrm{mg} /$ day, and up to $50 \%$ of cases with $>150 \mathrm{mg} /$ day. In women, menstrual disturbances and breast tenderness are described. In our cohort on spironolactone medication no major sexual adverse effects were observed which might have resulted in a change of medication.

\section{Discussion}

Blockade of the MR has been demonstrated to have a general renoprotective effect in patients with diabetes mellitus $(27,28,29,30)$ and with chronic kidney disease $(31,32,33)$ as indicated by a reduction of proteinuria. Although aldosterone excess as in PA is known to cause additional deleterious effects on the kidney (34), there are only a few studies investigating effectiveness of PA therapy on renal function. Ribstein et al. (11) reported a decrease in urinary albumin excretion after ADX in 25 PA patients who were followed up for 6 months. Sechi et al. (10) demonstrated that albuminuria declined during the initial 6-month treatment period in PA patients, and that restoration of normal albumin excretion was more common in PA than in EH. They also showed that PA patients did not differ in renal outcome (GFR and proteinuria) in comparison with patients with $\mathrm{EH}$ after a 9-year follow-up.

Our current prospective study is the largest cohort of PA patients so far analyzing renal function and confirms observations from a smaller cohort (10) and of our own retrospective data (3) regarding renal function and urinary albumin excretion. In our prospective study setting, we found a decline in GFR in early phase after initiation of the treatment. This observation has been explained by an aldosterone-induced hyperfiltration, which is abolished after treatment $(35,36,37)$, and was shown recently also for cystatin C-based estimations of

Table 3 Clinical and biochemical parameters in 119 patients with PA (47 females, 72 males) after initiation of treatment during long-term follow-up (study visits V1 and V2; cohort 2). Data are means \pm S.E.M.

\begin{tabular}{lcc}
\hline & V1 & V2 \\
\hline BMI (kg/m²) & $29.0 \pm 0.4$ & $29.1 \pm 0.5$ \\
Systolic/diastolic BP (mmHg) & $140 / 84 \pm 3 / 2$ & $139 / 83 \pm 3 / 2$ \\
24-h systolic/diastolic BP (mmHg) & $128 / 78 \pm 1 / 1$ & $130 / 79 \pm 1 / 1$ \\
24-h systolic/diastolic BP day (mmHg) & $132 / 81 \pm 1 / 1$ & $132 / 81 \pm 1 / 1$ \\
24-h systolic/diastolic BP night $(\mathrm{mmHg})$ & $119 / 71 \pm 1 / 1$ & $122 / 71 \pm 2 / 1$ \\
No. of antihypertensive drugs & $2.4 \pm 0.2$ & $2.6 \pm 0.2^{*}$ \\
Serum sodium (mmol/) & $138.9 \pm 0.3$ & $139.0 \pm 0.3$ \\
Serum potassium (mmol/l) & $4.16 \pm 0.04$ & $4.12 \pm 0.04$ \\
Urea (mg/dl) & $26.9 \pm 1.7$ & $28.9 \pm 2.8$ \\
Serum creatinine (mg/dl) & $1.09 \pm 0.03$ & $1.07 \pm 0.03$ \\
GFR (ml/min per 1.73 m ${ }^{2}$ ) & $67.5 \pm 1.9$ & $68.7 \pm 1.8$ \\
UAE/UCrea (mg/g) & $36.8 \pm 6.5$ & $33.0 \pm 8.6$ \\
\hline
\end{tabular}

$\mathrm{BP}$, blood pressure; UAE/UCrea, spot urine albumin excretion/ urinary creatinine. Normal ranges (SI units shown in brackets): sodium, 134-145 mmol//; potassium, $3.4-5.2 \mathrm{mmol} / \mathrm{l} ;$ urea, $10-50 \mathrm{mg} / \mathrm{dl}$; UAE/UCrea, $<20 \mathrm{mg} / \mathrm{g}$. ${ }^{\star} P<0.05$ compared with study visit $\mathrm{V} 1$. 
Table 4 Clinical and biochemical parameters in 188 patients with PA during long-term follow-up on study visit V1 depending on different therapies (cohort 2). Data are means \pm s.E.M.

\begin{tabular}{|c|c|c|c|c|}
\hline & ADX & Spiro & Epler & Others \\
\hline$n(\mathrm{~F} / \mathrm{M})$ & $86(52 / 34)$ & $65(25 / 40)$ & $18(2 / 16)$ & $19(9 / 10)$ \\
\hline Age (years) & $55.6 \pm 1.2$ & $64.3 \pm 1.2^{\|}$ & $58.8 \pm 2.3$ & $56.6 \pm 3.3$ \\
\hline $\mathrm{BMI}\left(\mathrm{kg} / \mathrm{m}^{2}\right)$ & $28.3 \pm 0.5$ & $28.8 \pm 0.6$ & $30.4 \pm 1.3$ & $28.4 \pm 1.0$ \\
\hline Duration of hypertension (years) & $17.8 \pm 1.5$ & $22.6 \pm 1.4^{\| \prime}$ & $17.4 \pm 2.9^{\mathrm{a}}$ & $15.6 \pm 3.79$ \\
\hline Time since diagnosis of PA (years) & $5.3 \pm 0.6$ & $5.9 \pm 0.5$ & $5.2 \pm 1.0$ & $4.6 \pm 1.1$ \\
\hline Dose MRA (mg/day) & & $63.5 \pm 5.8$ & $88.2 \pm 11.0$ & \\
\hline Systolic/diastolic BP (mmHg) & $133 / 84 \pm 2 / 1$ & $139 / 82 \pm 2 / 1$ & $142 / 88 \pm 3 / 2$ & $143 / 87 \pm 5 / 3$ \\
\hline 24-h systolic/diastolic BP $(\mathrm{mmHg})$ & $128 / 83 \pm 1 / 1$ & $132 / 78 \pm 2 / 1$ & $129 / 81 \pm 3 / 3$ & $137^{\dagger} / 79 \pm 4 / 2$ \\
\hline 24-h systolic/diastolic BP day ( $\mathrm{mmHg}$ ) & $131 / 82 \pm 1 / 1$ & $136 / 81 \pm 2 / 2$ & $132 / 85 \pm 3 / 3$ & $139 / 81 \pm 4 / 2$ \\
\hline 24-h systolic/diastolic BP night ( $\mathrm{mmHg}$ ) & $120 / 72 \pm 2 / 1$ & $124 / 72 \pm 2$ & $124 / 69 \pm 4 / 4$ & $129^{\dagger} / 74 \pm 4 / 1$ \\
\hline No. of antihypertensive drugs & $2.0 \pm 0.2$ & $2.2 \pm 0.2$ & $3.6 \pm 0.5^{\S, b}$ & $2.6 \pm 0.5$ \\
\hline Serum sodium $(\mathrm{mmol} / \mathrm{l})$ & $139.3 \pm 0.3$ & $138.9 \pm 0.3$ & $139.8 \pm 0.6$ & $139.7 \pm 0.7$ \\
\hline Serum potassium (mmol/l) & $4.25 \pm 0.06$ & $4.19 \pm 0.05$ & $3.97 \pm 0.09^{\star, a}$ & $3.81 \pm 0.09^{\# \# \#}$ \\
\hline Aldosterone (ng/l) & $92.4 \pm 10.3$ & $335.0 \pm 31.7^{\| \prime}$ & $436.2 \pm 77.0$ & $170.3 \pm 37.4^{\dagger}$ \\
\hline ARR & $11.7 \pm 2$ & $32.6 \pm 8.7^{\|}$ & $83.0 \pm 18.6^{\pi}$ & $49.5 \pm 28.0^{\dagger}$ \\
\hline Urea (mg/dl) & $31.3 \pm 2.3$ & $27.7 \pm 2.0$ & $35.4 \pm 3.5$ & $24.4 \pm 2.6$ \\
\hline $\operatorname{GFR}\left(\mathrm{ml} / \mathrm{min}\right.$ per $\left.1.73 \mathrm{~m}^{2}\right)$ & $72.0 \pm 2.8$ & $68.7 \pm 2.2$ & $73.9 \pm 4.5$ & $77.4 \pm 4.97$ \\
\hline UAE/UCrea $(\mathrm{mg} / \mathrm{g})$ & $28.3 \pm 6.1$ & $38.3 \pm 15.1$ & $73.5 \pm 28.3$ & $36.6 \pm 11.5$ \\
\hline
\end{tabular}

BP, blood pressure; ARR, aldosterone-renin ratio; UAE/UCrea, spot urine albumin excretion/urinary creatinine; ADX, adrenalectomy; Spiro, spironolactone; Epler, eplerenone; Others, other antihypertensive medication (of these 19 patients: $33.3 \%$ were on diuretics, $28.6 \%$ on beta-blockers, $33.3 \%$ on alpha-blockers, $33.3 \%$ on ACE inhibitors, 33.3\% on ATII antagonists, and $38.1 \%$ on calcium-channel blockers). Normal ranges (SI units shown in brackets): sodium, 134-145 mmol//; potassium, 3.4-5.2 mmol//; ARR, <20; urea, 10-50 mg/dl; UAE/UCrea, <20 mg/g. ${ }^{*,+} P<0.05 \mathrm{vs} \mathrm{ADX}$ group; ${ }^{\ddagger, \S} P<0.01$ vs ADX group; ${ }^{\# \# \# ~} P<0.001$ vs ADX group; $\|,{ }^{\uparrow} P<0.001$ vs $A D X$ group; ${ }^{\text {a }} P<0.05 ;{ }^{b} P<0.01$ vs Spiro group.

GFR (38). Previous studies have investigated this renal hyperfiltration with more accurate methods such as 24-h-creatinine clearance and radioisotopic clearance $(10,11)$. The observed glomerular hyperfiltration is associated with decreased intrarenal vascular resistance as assessed by duplex Doppler sonography (39). In a study by Catena et al. (40), the initial GFR and the decline in GFR after treatment were significantly lower in patients with a low pretreatment plasma aldosterone $(<225 \mathrm{pg} / \mathrm{ml})$. However, we did not find differences in GFR or urinary albumin excretion depending on pretreatment aldosterone levels. In our cohort with newly diagnosed PA patients (cohort 1), only age was correlated to renal outcome, whereas age, serum urea, and serum potassium levels were predictors for renal outcome in our PA patients already on treatment (cohort 2).

In our cohort 1 , we were able to show a significant decrease in serum sodium levels after initiation of therapy indicating the reversal of aldosterone-induced sodium-retaining effects and hypervolemia. The initiation of therapy affected especially the blood pressure during the night, which significantly dropped through therapy initiation.

PA patients of cohort 1 who underwent ADX showed marked improvement in blood pressure, serum potassium, and albumin excretion; however, patients who were started on medical treatment also showed improvements in these parameters, but they were not as strong as that in the adrenalectomized patients. These smaller improvements might be possibly due to the rather low average daily dose of $55 \mathrm{mg}$ spironolactone in cohort 1. Patients of cohort 2 received a higher average daily dose of spironolactone $(63.5 \pm 5.8 \mathrm{mg})$ and showed no differences in blood pressure or renal outcome to adrenalectomized patients. Our data suggest that at least $63.5 \mathrm{mg}$ of spironolactone daily might be necessary to sufficiently block aldosterone effects in PA patients; however, the exact dose needs to be adapted individually. This is supported by the finding from a Taiwanese study which failed to show significant changes in proteinuria after 1 year of spironolactone treatment with low doses (50 mg daily) (38).

Looking at the long-term outcome of PA patients in our prospective cohort 2 , it is remarkable that the GFR remained stable after such a long time of treatment supporting data from a previous smaller cohort (10). A slightly elevated UAE/Ucrea ratio was present in this cohort indicating some renal impairment. However, blood pressure remained in a normal range besides a slight increase in number of antihypertensive drugs. Furthermore, the UAE/Ucrea ratio remained low, indicating no further progress in renal damage. There is evidence that in PA, the advantage of blood pressure control is higher regarding renal function in comparison with patients with EH (10), probably due to reduction of high aldosterone levels or MR blockade, respectively, abolishing direct deleterious effects of aldosterone on kidney tissue.

In general our PA patients showed normal blood pressure levels and stable kidney function independent of the therapy they received. Considering previous studies, we are the first to investigate renal outcome depending on different therapeutic regimens in PA patients. Eplerenone is described to be as effective as spironolactone in reducing blood pressure in patients with BHA (41), whereas other studies claimed that the 
antihypertensive effect of spironolactone is significantly greater than that of eplerenone in PA patients (42). In the latter study, it was suggested that higher doses of eplerenone are needed to achieve the same effect as spironolactone. To our knowledge, our study is the first one investigating different PA therapies on renal function which included the MR antagonist eplerenone. Eplerenone seems as effective as ADX regarding renal function (GFR and UAE/Ucrea ratio). However, one noticeable difference was the lower serum potassium level compared with the other treatment groups. In a recent study, hyperkalemia occurred more often in spironolactone than in eplerenone-treated patients (42). Therefore, we believe that the eplerenone doses used in our study might still be too low to effectively block the MR. This is supported by the finding that the observed sufficient reduction in blood pressure levels and UAE/Ucrea ratio in the eplerenone group was only achieved due to a significantly higher number of other antihypertensive drugs. In addition, the MR antagonist spironolactone is also an androgen receptor antagonist and its effectiveness and superiority in blood pressure lowering might also be caused by the additional androgen receptor blockade $(43,44)$.

Patients who received no MR antagonist (e.g. due to spironolactone side effects) had significantly higher systolic blood pressure, lower serum potassium levels, and a higher number of antihypertensive drugs. This indicates the need of MR blockade in the therapy of PA.

Regarding lower serum potassium levels in the eplerenone group, further analysis is needed to evaluate if this subgroup has a disadvantage due to the lower potassium levels, e.g. resulting in a higher incidence of atrial fibrillation or renal cysts. In contrast to a previous report, where a significantly greater increase in serum aldosterone was observed in spironolactone- compared with eplerenone-treated PA patients after 4 months of therapy (42), we did not find a difference in aldosterone levels between these two groups in our study cohort. However, patients on spironolactone and eplerenone treatment had significant higher aldosterone levels than the ADX group, raising the problem of possible nonclassical actions due to these elevated aldosterone levels. However, no data are available yet regarding this topic. Limitations of our study were that we had no control cohort with EH for comparison.

In conclusion, we showed in a prospective study that distorted kidney function in PA is reversible after treatment initiation. Medical therapies with spironolactone or eplerenone seem to be as effective as ADX regarding renal function and blood pressure. However, our data suggest that eplerenone needs to be given in higher daily doses than spironolactone.

\section{Declaration of interest}

The authors declare that there is no conflict of interest that could be perceived as prejudicing the impartiality of the research reported.

\section{Funding}

This work was supported by the Else-Kroener-Fresenius-Foundation.

\section{Acknowledgements}

The authors would like to thank Franziska Hanusch, Gregor Hanslik, Christiane Friedrich, Kathrin Zopf (all Clinical Endocrinology, Berlin, Germany), and Susanne Schmid (University Hospital Munich).

\section{References}

1 Quinkler M \& Stewart PM. Treatment of primary aldosteronism. Best Practice and Research. Clinical Endocrinology and Metabolism 201024 923-932. (doi:10.1016/j.beem.2010.10.001)

2 Born-Frontsberg E, Reincke M, Rump LC, Hahner S, Diederich S, Lorenz R, Allolio B, Seufert J, Schirpenbach C, Beuschlein F et al. Cardio- and cerebrovascular comorbidites of hypo- and normokalemic primary aldosteronism: results of the German Conn's Registry. Journal of Clinical Endocrinology and Metabolism 2009 94 1125-1130. (doi:10.1210/jc.2008-2116)

3 Reincke M, Rump LC, Quinkler M, Hahner S, Diederich S, Lorenz R, Seufert J, Schirpenbach C, Beuschlein F, Bidlingmaier M et al. Risk factors associated with a low glomerular filtration rate in primary aldosteronism. Journal of Clinical Endocrinology and Metabolism 200994 869-875. (doi:10.1210/jc.2008-1851)

4 Rossi GP. Primary aldosteronism: a needle in a haystack or a yellow cab on fifth avenue? Current Hypertension Reports 20046 1-4. (doi:10.1007/s11906-004-0001-0)

5 Rossi GP, Bernini G, Desideri G, Fabris B, Ferri C, Giacchetti G, Letizia C, Maccario M, Mannelli M, Matterello MJ et al. Renal damage in primary aldosteronism: results of the PAPY Study. Hypertension $2006 \mathbf{4 8}$ 232-238. (doi:10.1161/01.HYP.0000 230444.01215.6a)

6 Born-Frontsberg E, Reincke M, Beuschlein F \& Quinkler M. Tumor size of Conn's adenoma and comorbidities. Hormone and Metabolic Research 200941 785-788. (doi:10.1055/s-0029-1224200)

7 Quinkler M, Born-Frontsberg E \& Fourkiotis VG. Comorbidities in primary aldosteronism. Hormone and Metabolic Research 201042 429-434. (doi:10.1055/s-0029-1243257)

8 Milliez P, Girerd X, Plouin PF, Blacher J, Safar ME \& Mourad JJ. Evidence for an increased rate of cardiovascular events in patients with primary aldosteronism. Journal of the American College of Cardiology 200545 1243-1248. (doi:10.1016/j.jacc.2005.01. 015)

9 Halimi JM \& Mimran A. Albuminuria in untreated patients with primary aldosteronism or essential hypertension. Journal of Hypertension 199513 1801-1802.

10 Sechi LA, Novello M, Lapenna R, Baroselli S, Nadalini E, Colussi GL \& Catena C. Long-term renal outcomes in patients with primary aldosteronism. Journal of the American Medical Association 2006 295 2638-2645. (doi:10.1001/jama.295.22.2638)

11 Ribstein J, Du Cailar G, Fesler P \& Mimran A. Relative glomerular hyperfiltration in primary aldosteronism. Journal of the American Society of Nephrology 200516 1320-1325. (doi:10.1681/ASN. 2004100878)

12 Catena C, Colussi G, Di Fabio A, Valeri M, Marzano L, Uzzau A \& Sechi LA. Mineralocorticoid antagonists treatment versus surgery in primary aldosteronism. Hormone and Metabolic Research 2010 42 440-445. (doi:10.1055/s-0029-1246185)

13 Sechi LA, Colussi G, Di Fabio A \& Catena C. Cardiovascular and renal damage in primary aldosteronism: outcomes after treatment. American Journal of Hypertension 201023 1253-1260. (doi:10.1038/ajh.2010.169)

14 Quinkler M, Zehnder D, Eardley KS, Lepenies J, Howie AJ, Hughes SV, Cockwell P, Hewison M \& Stewart PM. Increased expression of mineralocorticoid effector mechanisms in kidney biopsies of patients with heavy proteinuria. Circulation 2005112 1435-1443. (doi:10.1161/CIRCULATIONAHA.105.539122) 
15 Lam EY, Funder JW, Nikolic-Paterson DJ, Fuller PJ \& Young MJ. Mineralocorticoid receptor blockade but not steroid withdrawal reverses renal fibrosis in deoxycorticosterone/salt rats. Endocrinology 2006 147 3623-3629. (doi:10.1210/en.2005-1527)

16 Blasi ER, Rocha R, Rudolph AE, Blomme EA, Polly ML \& McMahon EG. Aldosterone/salt induces renal inflammation and fibrosis in hypertensive rats. Kidney International $2003 \mathbf{6 3}$ 1791-1800. (doi:10.1046/j.1523-1755.2003.00929.x)

17 Fujisawa G, Okada K, Muto S, Fujita N, Itabashi N, Kusano E \& Ishibashi S. Spironolactone prevents early renal injury in streptozotocin-induced diabetic rats. Kidney International 2004 66 1493-1502. (doi:10.1111/j.1523-1755.2004.00913.x)

18 Sun GP, Kohno M, Guo P, Nagai Y, Miyata K, Fan YY, Kimura S, Kiyomoto H, Ohmori K, Li DT et al. Involvements of rho-kinase and TGF- $\beta$ pathways in aldosterone-induced renal injury. Journal of the American Society of Nephrology 200617 2193-2201. (doi:10.1681/ASN.2005121375)

19 Toyonaga J, Tsuruya K, Ikeda H, Noguchi H, Yotsueda H, Fujisaki K, Hirakawa M, Taniguchi M, Masutani K \& Iida M. Spironolactone inhibits hyperglycemia-induced podocyte injury by attenuating ROS production. Nephrology, Dialysis, Transplantation 201126 2475-2484. (doi:10.1093/ndt/gfq750)

20 Chen C, Liang W, Jia J, van Goor H, Singhal PC \& Ding G. Aldosterone induces apoptosis in rat podocytes: role of PI3-K/ Akt and P38MAPK signaling pathways. Nephron. Experimental Nephrology 2009113 e26-e34. (doi:10.1159/000228080)

21 Reincke M, Meisinger C, Holle R, Quinkler M, Hahner S, Beuschlein F, Bidlingmaier M, Seissler J \& Endres S. Is primary aldosteronism associated with diabetes mellitus? Results of the German Conn's Registry. Hormone and Metabolic Research 201042 435-439. (doi:10.1055/s-0029-1246189)

22 Fischer E, Beuschlein F, Bidlingmaier M \& Reincke M. Commentary on the Endocrine Society Practice Guidelines: consequences of adjustment of antihypertensive medication in screening of primary aldosteronism. Reviews in Endocrine \& Metabolic Disorders 201112 43-48. (doi:10.1007/s11154-011-9163-7)

23 Levey AS, Stevens LA, Schmid CH, Zhang YL, Castro AF, Feldman HI, Kusek JW, Eggers P, Van Lente F, Greene T et al. A new equation to estimate glomerular filtration rate. Annals of Internal Medicine 2009150 604-612.

24 Botev R, Mallie JP, Wetzels JF, Couchoud C \& Schuck O. The clinician and estimation of glomerular filtration rate by creatininebased formulas: current limitations and Quo Vadis. Clinical Journal of the American Society of Nephrology 2011 6 937-950. (doi:10.2215/CJN.09241010)

25 Funder J, Carey R, Fardella C, Gomez-Sanchez C, Mantero F, Stowasser M, Young W \& Montori VM. Case detection, diagnosis, and treatment of patients with primary aldosteronism: an Endocrine Society Clinical Practice Guideline. Journal of Clinical Endocrinology and Metabolism 200893 3266-3281. (doi:10.1210/jc.2008-0104)

26 Vonend O, Ockenfels N, Gao X, Allolio B, Lang K, Mai K, Quack I, Saleh A, Degenhart C, Seufert J et al. Adrenal venous sampling: evaluation of the German Conn's Registry. Hypertension 201157 990-995. (doi:10.1161/HYPERTENSIONAHA.110.168484)

27 Mehdi UF, Adams-Huet B, Raskin P, Vega GL \& Toto RD. Addition of angiotensin receptor blockade or mineralocorticoid antagonism to maximal angiotensin-converting enzyme inhibition in diabetic nephropathy. Journal of the American Society of Nephrology 200920 2641-2650. (doi:10.1681/ASN.2009070737)

28 Sato A, Hayashi K, Naruse M \& Saruta T. Effectiveness of aldosterone blockade in patients with diabetic nephropathy. Hypertension 2003 41 64-68. (doi:10.1161/01.HYP.0000044937.95080.E9)

29 Schjoedt KJ, Rossing K, Juhl TR, Boomsma F, Tarnow L, Rossing P \& Parving HH. Beneficial impact of spironolactone on nephrotic range albuminuria in diabetic nephropathy. Kidney International 200670 536-542.

30 Epstein M, Williams GH, Weinberger M, Lewin A, Krause S, Mukherjee R, Patni R \& Beckerman B. Selective aldosterone blockade with eplerenone reduces albuminuria in patients with type 2 diabetes. Clinical Journal of the American Society of Nephrology 20061 940-951. (doi:10.2215/CJN.00240106)

31 Epstein M. Aldosterone receptor blockade and the role of eplerenone: evolving perspectives. Nephrology, Dialysis, Transplantation 200318 1984-1992. (doi:10.1093/ndt/gfg339)

32 Bianchi S, Bigazzi R \& Campese VM. Antagonists of aldosterone and proteinuria in patients With CKD: an uncontrolled pilot study. American Journal of Kidney Diseases $2005 \mathbf{4 6}$ 45-51. (doi:10.1053/j.ajkd.2005.03.007)

33 Furumatsu Y, Nagasawa Y, Tomida K, Mikami S, Kaneko T, Okada N, Tsubakihara Y, Imai E \& Shoji T. Effect of reninangiotensin-aldosterone system triple blockade on non-diabetic renal disease: addition of an aldosterone blocker, spironolactone, to combination treatment with an angiotensin-converting enzyme inhibitor and angiotensin II receptor blocker. Hypertension Research 200831 59-67. (doi:10.1291/hypres.31.59)

34 Fourkiotis VG, Hanslik G, Hanusch F, Lepenies J \& Quinkler M. Aldosterone and the kidney. Hormone and Metabolic Research 2012 44 194-201. (doi:10.1055/s-0031-1295461)

35 Briet M \& Schiffrin EL. Aldosterone: effects on the kidney and cardiovascular system. Nature Reviews. Nephrology 20106 261-273. (doi:10.1038/nrneph.2010.30)

36 Nishimura M, Uzu T, Fujii T, Kuroda S, Nakamura S, Inenaga T \& Kimura G. Cardiovascular complications in patients with primary aldosteronism. American Journal of Kidney Diseases 1999 33 261-266. (doi:10.1016/S0272-6386(99)70298-2)

37 Wu VC, Yang SY, Lin JW, Cheng BW, Kuo CC, Tsai CT, Chu TS, Huang KH, Wang SM, Lin YH et al. Kidney impairment in primary aldosteronism. Clinica Chimica Acta $2011 \mathbf{4 1 2} 1319-1325$. (doi:10.1016/j.cca.2011.02.018)

38 Wu VC, Kuo CC, Wang SM, Liu KL, Huang KH, Lin YH, Chu TS, Chang HW, Lin CY, Tsai CT et al. Primary aldosteronism: changes in cystatin C-based kidney filtration, proteinuria, and renal duplex indices with treatment. Journal of Hypertension 201129 1778-1786. (doi:10.1097/HJH.0b013e3283495cbb)

39 Sechi LA, Di Fabio A, Bazzocchi M, Uzzau A \& Catena C. Intrarenal hemodynamics in primary aldosteronism before and after treatment. Journal of Clinical Endocrinology and Metabolism 2009 94 1191-1197. (doi:10.1210/jc.2008-2245)

40 Catena C, Colussi G, Nadalini E, Chiuch A, Baroselli S, Lapenna R \& Sechi LA. Relationships of plasma renin levels with renal function in patients with primary aldosteronism. Clinical Journal of the American Society of Nephrology $2007 \quad 2 \quad 722-731$. (doi:10.2215/CJN.00050107)

41 Karagiannis A, Tziomalos K, Papageorgiou A, Kakafika AI, Pagourelias ED, Anagnostis P, Athyros VG \& Mikhailidis DP. Spironolactone versus eplerenone for the treatment of idiopathic hyperaldosteronism. Expert Opinion on Pharmacotherapy 20089 509-515. (doi:10.1517/14656566.9.4.509)

42 Parthasarathy HK, Menard J, White WB, Young WF Jr, Williams GH, Williams B, Ruilope LM, McInnes GT, Connell JM \& MacDonald TM. A double-blind, randomized study comparing the antihypertensive effect of eplerenone and spironolactone in patients with hypertension and evidence of primary aldosteronism. Journal of Hypertension 201129 980-990. (doi:10.1097/ HJH.Ob013e3283455ca5)

43 Hofmann PJ, Michaelis M, Gotz F, Bartel C, Kienitz T \& Quinkler M. Flutamide increases aldosterone levels in gonadectomized male but not female Wistar rats. American Journal of Hypertension 2012 25 697-703. (doi:10.1038/ajh.2012.21)

44 Michaelis M, Hofmann PJ, Gotz F, Bartel C, Kienitz T \& Quinkler M. Sex-specific effects of spironolactone on blood pressure in gonadectomized male and female Wistar rats. Hormone and Metabolic Research 201244 291-295. (doi:10.1055/s-0031-1299774)

Received 23 July 2012

Revised version received 11 September 2012

Accepted 2 October 2012 